\title{
The Application of HHT Method Used in the Anchor Bolt Testing Signal
}

\author{
Linsong Wang ${ }^{1}$, Junmin Wang ${ }^{2}$,Mingzhang Luo $^{2}$, Chao Chen ${ }^{1}$, Qiuge Wang ${ }^{1}$, Kun Wang ${ }^{3}$ \\ 1. Institute of Geophysics \& Geomatics. China University of Geosciences. Wuhan, 430074, China \\ 2. Yangtze University, Jingzhou, China \\ 3. Faculty of Engineering, China University of Geosciences. Wuhan, China \\ wanglinsong123@yahoo.cn,wjm8510@163.com
}

\begin{abstract}
When varying degrees defects existing in the anchorage system, energy decreasing and frequency mutation can be caused during the acoustic waves propagation. We can get the instantaneous frequency of the signal by processing the analysis of anchor bolt testing signal using the method of HHT (Hilbert-Huang Transform), then we derive the accurate expression of its frequency varying over time. In addition, we can obtained the Time-Frequency distribution of the energy in three-dimensional space (which also known as the distribution of Hilbert) on the basis of the expression. The analysis results of the simulated signal and actual signals (measurement signals) indicated that the HHT method can collect the effective information Comparatively accurate thus validate that this method can apply to anchor bolt testing signals with variable energy and frequency preferably.
\end{abstract}

\section{Keywords-Anchor bolt, HHT, Time-Frequency Analysis}

\section{INTRODUCTION}

During the propagation of the audiofrequency stress waves inspired transiently in the anchorage system, the variation of anchorage system generalized wave impedance can arouse in response to the boundary effect of stress wave. When the cross section or material property in the pole changes, there will be Transmission and reflection of the incident waves in the section will take place when the cross section or material property in the pole varation.. The amplitudes of reflection and transmission waves have relation to the relative variation degree of the cross section and wave impedance. As the difference of the three wave impedances is not that big when it is poured homogeneously and compacted with anchor pole , mortar and surrounding rock, there are big parts of energies transmission coming out of it and only a few of the energies reflect back. When it is not poured homogeneously or compacted with mortar, there will be strong differences of wave impedances in the space between mortars, which show that a reflection wave signal was added to the original signal which has stranger reflection wave energies as a result. By analyzing the energy relationship between the reflection waves and transmission waves, compaction rate of the anchor bolt age system can be judged. At the same time, when the stress wave meets the anchoring defects, the original vibration changes and shows that there are an abrupt change of frequency and phase on the anchoring defects. Therefore, the position of the anchoring defects can be judged by analyzing the instantaneous frequency variation position of the reflection waves.
Most of the anchor bolt test signals can be nonlinear and unsteady signals, the existed signal processing methods (e.g. FFT)can hardly meet the demands of the nonlinear and unsteady signals processing. In 1998, a method of signal processing named Hilbert-Huang Transform (HHT) was brought up by Chinese American Norden E. Huang of National Aeronautics and Space Administration (NASA), which is considered as a big breakthrough of the linear and steady-state spectrum analysis based on Fourier transformation[1]. The HHT is regarded as an important revolution in the signal processing field, which has clear physical meanings with nonlinear and unsteady signals processing .By this way, the signals' energy-frequency-time distribution characteristics are derived .So the HHT is an adaptive signal processing method.

This paper focuses on the anchor bolt test signals' processing and analysis based on the HHT method. First, signals are processed with an adaptive decomposition of Empirical Mode Decomposition (EMD) and then the low frequency signals that have nothing to do with the anchor bolt test should be eliminated on the basis of these decomposed frequency characteristics from low to high. At the same time, the signals instantaneous power map can be available by calculating the remaining energies using HHT. And then, the effective information of the original signals will be obtained accurately.

\section{HHT(HILBERT-HUANG TRANSFORM)}

Hilbert-Huang Transform (HHT) is designed by U.S. citizen Chinese NE. Huang according to the modern wellknown mathematician Hilbert's mathematics theory in 1998, which is a signal processing method aimed at analyzing nonlinear and unsteady signals.

In 1999, Huang acquired some improvements in this method. The improved one was composed of two parts: Empirical Mode Decomposition (EMD) and Hilbert analysis of spectrum (HAS).The HHT is a essentially method used in stably processing signals which means to decompose the time signal into numbers of Intrinsic Mode Functions (IMF) by the mode of Empirical Mode Decomposition (EMD) and then the time-frequency distribution can be obtained after the HHT processing to each of the decomposed Intrinsic Mode Functions (IMF). The Hilbert spectrum derived by this method has a very high time-frequency resolution and it has overcome some kinds of drawbacks that exist in the former signal processing methods based on Fourier analysis. Moreover, the Intrinsic Mode Functions (IMF) parameters 
obtained from the signals decomposition used EMD has a clearly physical meaning. This method can not only make the signal decomposition with uniqueness but also make the signal decomposition having good localization characteristics both on time and frequency fields. Once the signal was decomposed completely, the signal could be reconstructed flexibly considering the problem to be solved [2-4].

EMD is basically a process of separating IMF from the complicated signal, which can also be called the sifting process. Generally, as the original real signal is complex, the EMD method is on the assumption that each of the signals are composed of different kinds of IMF and each of the IMF can be linear or nonlinear and have the same number of extreme points and zero-crossing points which means that they distribute symmetrically in the up and down area of the horizontal axis. The IMF can be used under two conditions:(1) the number of extreme points and zerocrossing points should be almost the same (or by a difference of 1, at most); (2) the mean value of the two envelops composed of the local maximum points and local minimum points should be 0 at any points.

As each of the IMF parameters is a data set that represents a group of characteristic scales, the whole process can be regarded as a superposition of processes during which the original data set is divided into different characteristic waves. As a result, the original data set can be recovered by converse superposition of all the IMF parameters. Thus it can be seen that the EMD method needs the position of local extreme value instead of a mean value or a zero frame of reference. The zero frames of reference of every parameter can be realized by the process of screening. The trouble of removing the mean value aroused by the relatively big DC (direct current) terms of the not zero mean value data is avoided by the EMD method. Therefore, the EMD method is decomposed on the basis of the original signals and the obtained IMF parameters numbers would be limited. What's more, each parameters has completeness and approximately orthogonality .Therefore, this decomposition is a method of uniqueness and adaptability[5-6].

After the EMD processing with original signals, frequency of each IMF obtained fells in turn. The trend line is the sign of the trend of original signals decomposition and represents the whole trend of signals with little energy. In practically, flexible reconstitution can be done with the signals to achieve effects of LPF, HPF and BPF which are based on characteristics of parameter frequencies' ranging from high to low. The instantaneous spectrum of each IMF can be derived from the EMD decomposed IMF processed with Hilbert transformation. A new kind of time-frequency description -Hilbert spectrum can be obtained b synthesizing all the IMF parameters' instantaneous spectrum. Hilbert transformation is a kind of linear transformation in which the obtained instantaneous spectrum has rather clear physical significance and the high or low frequency values not existing actually caused by Fourier transformation can be filtered.

\section{APPLICATION}

For verifying the efficiency of this method, an ideal signal is designed which is superimposed of simulated signals with three different time periods and different frequencies. The signals expressions are below respectively:

$$
\begin{aligned}
& A_{1}=e^{-0.01 \times n_{1} \times \Delta t_{1}} \times \cos \left(2 \pi \times f_{1} \times n_{1} \times \Delta t_{1}\right) \\
& A_{2}=0.5 \times \cos \left(2 \pi \times f_{2} \times n_{2} \times \Delta t_{2}\right) \\
& A_{3}=0.8 \times \cos \left(2 \pi \times f_{3} \times n_{3} \times \Delta t_{3}\right)
\end{aligned}
$$

In the equations $f$ is the signal frequency, $f_{1}=1 / 32 \mathrm{~Hz}, f_{2}=10 \mathrm{~Hz}$; The parameter $n$ is the sampling number $n_{1}=1,2, \cdots, 512$

$n_{2}=100,101, \cdots, 512, n_{3}=200,201, \cdots, 512 ; \Delta t$ is the sampling interval, $\Delta t_{1}=\Delta t_{2}=\Delta t_{3}=1 \mathrm{~s}$.

Making a processing of HHT after calculating the sum of $\mathrm{A} 1 、 \mathrm{~A} 2$ and $\mathrm{A} 3$, the results are shown in figure.1.In this figure, two kinds of ideal signals with different frequencies and amplitudes were added in the time 100s 、200s respectively to the cosine wave(Figure.1-(a)) with energy exponent attenuation during the whole period. On the basis of superposition, the simulated signal (Figure.1-(b)) with energy attenuation and abrupt change features can be obtained and its characteristics can satisfied the condition of exponent relationship between the anchor length in practical bolt signal test and stress wave energy attenuation. And it can also satisfied the characteristic that there are frequency of mutation in the weak place of anchor, and so on. Four IMF parameters IMF1、IMF2、IMF3、IMF4, and residual parameter are derived after the decomposition of the added signals used EMD method, see Figure.1-(c). From the figure, the first two parameters IMF1、IMF2 include the energy exponent attenuation and basic abrupt change information of the original added signals. IMF3、 IMF4 and residual are regarded as a characteristic of the relatively low frequency and entirety trend of the signals. Considering the characteristic of the frequency obtained using EMD method changing from high to low, IMF3、IMF4 and residual as low frequency information will not be included of the Hilbert transformation in the later period.

The 3D Hilbert spectrum (see Figure.1-(d)) of timefrequency-amplitude of processed IMF1 、 IMF2 with Hilbert transformation shows clearly that HHT method has a strong responding to the signal abrupt change. There are corresponded frequencies abrupt points both at the synthetic signal points of $100 \mathrm{~s}$ and $200 \mathrm{~s}$. Then the signal energy attenuation trend with the time changes and its disappearing after the time point 400 s can be seen clearly from the changes of the amplitude values represented of colors. It is indicated that the instantaneous frequency signal can be a good correspondence in each anomaly time points with the original added signals by the processing and the analysis of the simulated signal.

In order to indicate the processing efficiency and correctness of the HHT method used in the anchor bolt signal test, an actual signal got from the process of slope 
reparation and consolidation engineering in the highway section is analyzed in the following. The practical anchor length is $5-17 \mathrm{~m}$ and the data sampling use the LX-10E anchor quality testing technique. The actual signal and the result processed with EMD method are expressed in the figure in which the horizontal ordinates are points number that is multiplied by the sampling interval (in this paper it is $2 \mu s$ ). As a result, the wave transformed time can be obtained with a velocity value of $5000 \mathrm{~m} / \mathrm{s}$ when it transforms through steels. Figure.2-(a) shows a bad regularity result of the original signal wave form and the reflected wave energy declines gradually. To the parameters derived from the EMD decomposition, IMF1、IMF2 kept the basic information of energy decreasing and mutation of the original signal. So, the parameters with relatively low frequency and residuals after the IMF2 are not included in the Hilbert transformation.Then the 3D Hilbert spectrum of time -frequency -amplitude is shown in Figure. 3.

In the anchorage system, the sound wave transmitted by the ending part of the anchor spreads to the surrounding and there would be incidence, reflection and transmission in the section of anchor pole, mortar and surrounding rock. When the anchorage system is homogeneous and compacted, most of the stress wave energy leak out into the surrounding rocks and only a few of the energies reflect back. What's more, when mortar is not mixed homogeneous and compacted, there would be different degrees of varying wave impedance with a representation that the original signal is superposed by reflection signal which induced sudden changes of frequency and phase [7] with a different frequency. Through the Figure.3, the signal's high frequency periods are mainly focused on $0-5 \mathrm{KHz}$ according to the changing characteristic of the frequency and amplitude related to time. It is apparently that the frequency changing and the energy amplitude attenuation at about the $7.5 \mathrm{~m}$ (arrow position at point number 750), based on which it can be conclude as the reflection information of the anchor bottom. As there are frequency sudden changing information in the position of 1.5 $\mathrm{m}$ (point number150)、2.7 $\mathrm{m}$ (point number270)and 4.4 $\mathrm{m}$ (point number440), it can be predicted that the three parts has defects based on different frequencies can be aroused by the defects' position, but the defects' range is not so big. So synthesizing the anchor length and the defect position it can be regarded as a general qualified anchor.

\section{CONCLUSION}

HHT is a kind of not-steady analysis method of decomposing the signal into the sum of some limited IMF based on EMD. Then the instantaneous frequency of signal can be obtained through a Hilbert transformation and the precise expression of frequency changing with time can also be got. On the basis of these results, the distribution of energy (Hilbert spectrum) in the 3D time frequency space can be calculated as a result. Results of simulated signal and real signal analysis verified that this method can be used in anchor bolt test signal with changing energy and frequency.

As a well developed method in recent years, there are still many problems on HHT that are not resolved yet, such as interpolation methods, end effect and so on. In this paper, it is just an attempt of using the HHT method in the feature information extraction from the anchor test signal. As the work in this paper is only the first step of research, there are still some text phenomena that need to further theory interpretation and systemic study, such as the influence of absorption and scattering attenuation to the sound wave's transmission through the bolt, the relationship of Hilbert spectrum frequency, amplitude and the transmission of the sound wave in the bolt , and so on.

\section{ACKNOWLEDGMENT}

This work was supported in part by the National Nature Science Fund of China under Grants 40727001 and 40774073.

\section{REFERENCES}

[1] N. E. Huang, Z. Shen, S. R. Long, et al. The empirical mode decomposition and Hilbert spectrum for nonlinear and nonstationary time series analysis, Proc. Roy. Soc. London A, 1998, 454, PP:903905

[2] Norden E. Huang; Zheng Shen; Steven R. Long. A new view of nonlinear waterwaves: The Hilbert Spectrum, Annu. Rev. Fluid Meeh[J], 1999, 31, PP:417-457

[3] Zhaohua $\mathrm{Wu}$; Norden E. Huang. A study of the characteristics of white noise using the empiriealmod decomposition method, roc. Roy. Soc. London A, 2004, 460, PP:1594-1611

[4] Norden E. Huang; Man-Li C. Wu; Steven R. Long; Samuel S. P. Shen; WenDong Qu; Per Gloersen; Kuang L. Fan. A confidence limit for the empirical mode Decomposition and Hilbert spectral analysis, Proc. Roy. Soe. London A, 2003, 459, PP:2317-2345

[5] P. Flandrin; G. Rilling and P. Goncalves. Empirical Mode Decomposition as a filter bank, IEEE Sig. Proc. Lett.[J], 2004, 11(2), PP:112-114

[6] P. Flandrin and P. Gonealves. Empirical Mode Decompositions as data driven wavelet-like expansio [Dissertation], on Wavelets and Multires, Info. Proe, 2004

[7] Junming Wang; Yiqun Chen; Hua Chen. Research on anchor bar quality nondestructive testing technique on highway. Progress in geophysics[J], 2004, 19(4),PP:782-785 


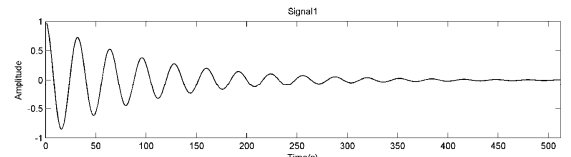

(a) $A_{1}$

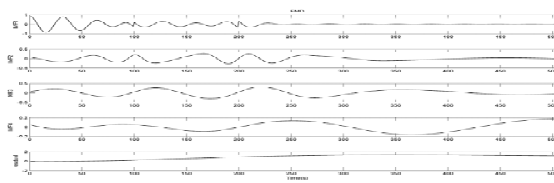

(c) The EMD of $A_{1}+A_{2}+A_{3}$

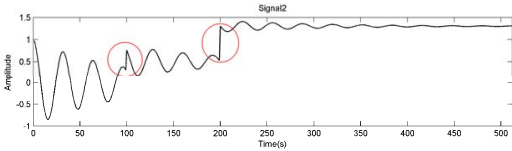

(b) $A_{1}+A_{2}+A_{3}$

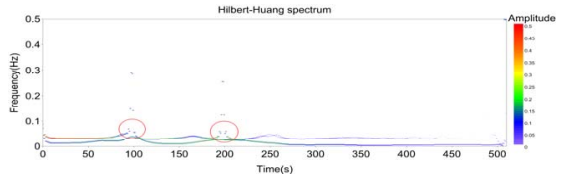

(d) Hilbert-Huang spectrum

Figure 1. Simulated signal, parameters and radiuses processed by EMD method and the Hilbert-Huang spectrum

(a) simulated signal ${ }^{A_{1}}$; (b) simulated signal $A_{1}+A_{2}+A_{3}$; (c) The EMD of $A_{1}+A_{2}+A_{3}$;(d) The

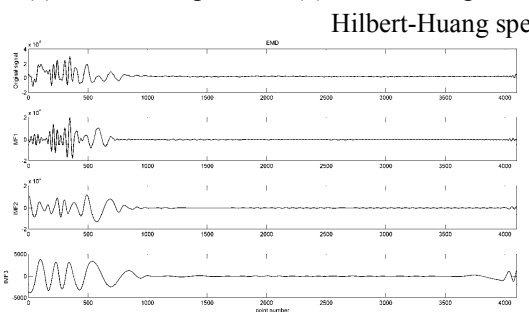

(a) Original signal and IMF1-IMF3

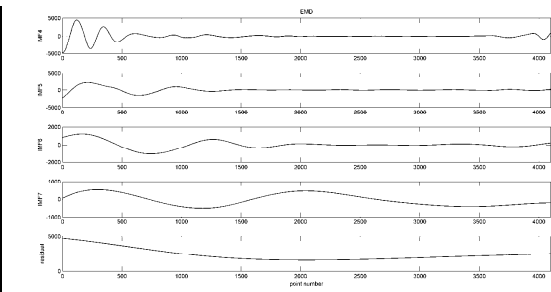

(b) IMF4-IMF7 and residual

Figure 2. Original actual signal, IMF parameters and radiuses processed by EMD method

(a) Original signal and IMF1-IMF3; (b) IMF4-IMF7 and residual
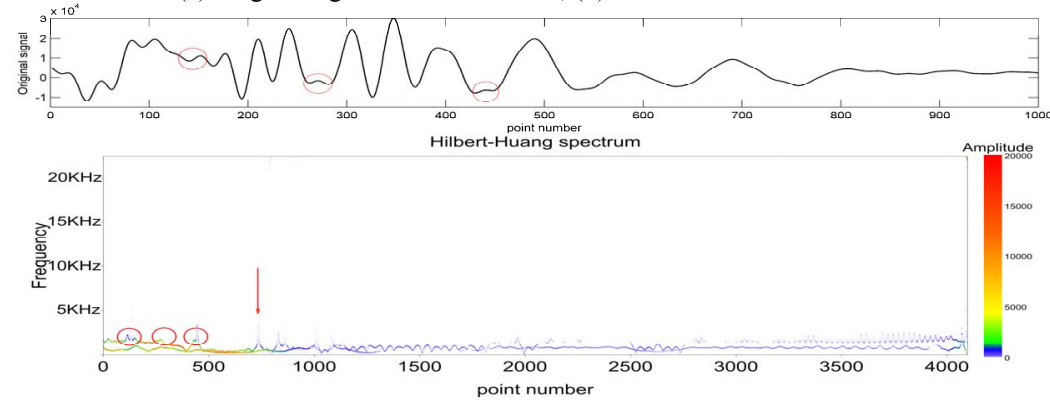

Figure 3. The Hilbert-Huang spectrum of IMF1+IMF2 